\title{
Ville fossile
}

(Suite poétique extraite de La Voyageuse, un recueil inédit)

\author{
Andrée Lacelle
}

\section{Fossil City}

The City is deconstructed as its fossilized quarters come to life. (In the shadowy zone, a woman makes a break, changes the subject). An exiled era advances ecstatically toward the declining City from the wild background, the outskirts where totalities are dissolved in absolute fragmentation. A gleaming figure moves across the cloistered air in long strides. (The hand of a woman, waving, is caught by a ray oflight). Sepia seeps into the surveyed field. The eternal present is traversed by a woman on the look-out.

Au départ de la voyageuse

il n'y a pas foule

à la porte du phare

veille la douce force de l'amour

Au rythme de la distance

elle avance dans l'entre-deux

recollant les séquences de l'époque de veille

quant toutes choses fugaces s'exposent loin des routes immédiates

II

Elle débarque au centre du monde

où vus d'en bas

de lumineux plissements montagneux

se confondent aux contours des nuages

et lui parvient l'impression palpable des surfaces éloignées

sans fin en état de recul

aux abords d'une ville sans porte

en deçà de toute fusion des antipodes

en territoire quotidien 
à sa lisière

l'écart désormais éphémère

échappe au temps accéléré

à l'essor illusoire de la Cité en son déclin

répond le délire de l'apesanteur

de l'œil et de l'arme

de la prouesse d'une ère en exil

III

Le relief sauvage de la ville en son centre absent oscille

les débris de la ville projetés

dans l'ampleur ouverte d'une contrée inerte

déconstruite en terrain improbable

ville fossile parmi tours et détours

déploie sa dimension perdue

IV

En zone d'ombre

femme lente et longue

quitte le domicile rompu

sillonne les espaces inséparables

franchit la demeure pénétrable

peuple le temps profond

femme lueur gravite

lutte mouvante

risque le délire

arpente l'air claustral

toise l'axe de la figure idéale

V

un rai de lumière traverse les mains d'une femme saluant

au pays qui se tait coule sépia sur champ cadastral

ultime repère point par point

femme vigie traverse l'éternel présent 